\title{
DISCRETE ORDERED SETS WHOSE COVERING GRAPHS ARE MEDIAN
}

\author{
HANS - J. BANDELT
}

\begin{abstract}
As is well known the covering graph (= Hasse diagram) of any median semilattice is a median graph, and every median graph is the covering graph of some median semilattice. The purpose of this note is to prove that an ordered set is a median semilattice whenever (i) no interval contains an infinite chain, (ii) each pair of elements is bounded below, and (iii) the covering graph is median.
\end{abstract}

The covering graphs (alias undirected Hasse diagrams) of finite distributive lattices have been characterized in several ways; see $[\mathbf{1}, \mathbf{2}, \mathbf{4}]$. For instance, S. P. Avann [2] proved the following result: a finite graph $G$ is the covering graph of a finite distributive lattice if and only if $G$ is a median graph with two distinguished vertices 0 and 1 such that every vertex in $G$ lies on a shortest path joining 0 and 1 . Recall that a median graph is a (not necessarily finite) connected graph in which for any three vertices $u, v, w$ there is exactly one vertex $x$ (the median of $u, v, w$ ) such that $d(u, v)=d(u, x)+d(x, v), d(v, w)=d(v, x)+d(x, w)$, and $d(u, w)=$ $d(u, x)+d(x, w)$, where $d$ is the distance function of the graph. Every median graph is the covering graph of a median semilattice with least element $a$, where $a$ is any fixed vertex: for vertices $u$ and $v$ put $u \leqslant_{a} v$ if $d(a, v)=d(a, u)+d(u, v)$; see $[2,6]$; cf. [5]. Median semilattices are close to distributive lattices and are defined as follows. A meet-semilattice is called a median semilattice if every principal ideal is a distributive lattice and any three elements have an upper bound whenever each pair of them is bounded above. The covering graph of a median semilattice $S$ is a median graph provided that $S$ is discrete, i.e. all intervals are finite; see [2]. In general, an ordered set $P$ is called discrete if all bounded chains in $P$ are finite. Then from [2] one can derive the following result: a discrete lattice $L$ with 0 is distributive if and only if the covering graph of $L$ is median. In this theorem the requirement that $L$ have a least element can be dropped; see [3]. Here we prove a more general result.

THEOREM. Let $P$ be a discrete ordered set in which any two elements are bounded below. Then $P$ is a median semilattice if and only if the covering graph of $P$ is median.

COROLlaRY. $A$ discrete ordered set $P$ is a distributive lattice if and only if (i) any two elements are bounded below and bounded above, and (ii) the covering graph of $P$ is median.

Received by the editors July 14, 1981 .

1980 Mathematics Subject Classification. Primary 06A10, 06A12, 06D99; Secondary 05C99.

Key words and phrases. Discrete ordered set, covering graph, median semilattice, distributive lattice, median graph. 


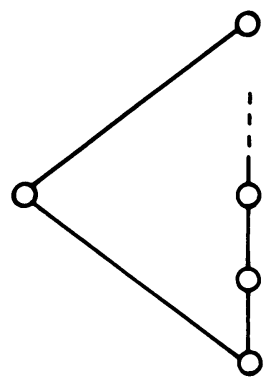

FIGURE 1

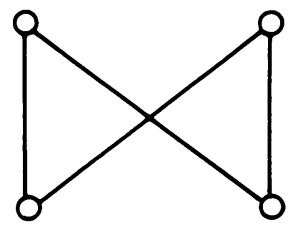

FIGURE 2

The conditions of the Theorem cannot be relaxed as is shown by Figure 1 [3] and Figure 2.

Henceforth the covering graph of a discrete ordered set $P$ is denoted by $C(P)$. The segment of two vertices $u$ and $v$ in a connected graph is the set $u \circ v$ of all vertices $x$ which lie on a shortest path joining $u$ and $v$; that is, $d(u, x)+d(x, v)=d(u, v)$.

Lemma. Let $P$ be a discrete ordered set such that $C(P)$ is a median graph. Then for any $a<b$ in $P$, the interval $[a, b]=\{\hat{x} \in P \mid a \leqslant x \leqslant b\}$ coincides with the segment $a \circ b$ of $C(P)$.

Proof. (i) To prove that $[a, b] \subseteq a \circ b$ for $a<b$, one has to check that any maximal chain between $a<b$ in the ordered set $P$ is a shortest path joining $a$ and $b$ in the graph $C(P)$. This is, in fact, not difficult to show by using induction on the length of the chain; see the first part of the proof of Theorem 4.5 in [3].

(ii) Suppose that $a<b$ in $P$ and $x \circ y \subseteq[x, y]$ for all $x<y$ in $P$ with $d(x, y)<$ $d(a, b)$. Pick any element $v$ of $[a, b]$ which is covered by $b$. Let $a, z_{1}, z_{2}, \ldots, z_{n-1}, w, b$ be any shortest path joining $a$ and $b$. Since $v \in a \circ b$ by (i), the median $u$ of $a, v, w$ belongs to $a \circ v \cap a \circ w \subseteq[a, v] \cap[a, w]$. Then either $u=v=w$ or $d(b, u)=2$, whence $w \in u \circ b \subseteq[u, b]$ and thus $a \leqslant w<b$. By assumption, the subpath $a, z_{1}, \ldots, z_{n-1}, w$ is contained in $[a, w]$, and therefore the whole path is contained in $[a, b]$. This proves the required inclusion $a \circ b \subseteq[a, b]$.

Proposition. Let $P$ be a discrete ordered set such that $C(P)$ is a median graph. Then every interval of $P$ is a finite distributive lattice.

Proof. In view of the Lemma every interval $[a, b]$ of $P$ is a segment of the graph $C(P)$. Therefore the covering graph of $[a, b]$ is a median graph. Hence by Avann's theorem (or [3, Theorem 4.5]) $[a, b]$ is a (finite) distributive lattice provided that $[a, b]$ is a lattice. Suppose by way of contradiction that there exists an interval $[a, b]$ of $P$ which is not a lattice. Then choose $v, w, x, y \in[a, b]$ such that $v$ and $w$ are distinct minimal upper bounds of $x$ and $y$. By the Lemma the median $u$ of $a, v, w$ is a lower bound of $v$ and $w$. Further, we get that $x, y \in a \circ v \cap a \circ w=a \circ u$, whence $u$ is an upper bound of $x$ and $y$. This contradicts the minimality of $v$ and $w$, and we are done. 


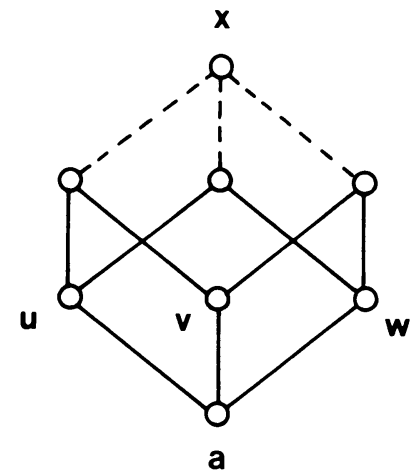

FIGURE 3

Proof of The Theorem. Since any two elements of $P$ are bounded below, we infer from the Proposition that $P$ is a meet-semilattice in which every principal ideal is a distributive lattice. Assume that $P$ is not a median semilattice. Then choose $u, v, w \in P$ such that (1) $u, v, w$ have no common upper bound, (2) $u \vee v, u \vee w$, $v \vee w$ exist, and (3) for $a=u \wedge v \wedge w$, the ternary distance $n=d(a, u)+d(a, v)$ $+d(a, w)$ is minimal with respect to (1) and (2). If, say, $u$ does not cover $a$, then pick any $t$ with $a<t<u$. Clearly, $t$ is the meet of $u, t \vee v$, and $t \vee w$. Since $t \vee v \vee w$ exists by (3), the elements $u, t \vee v, t \vee w$ are pairwise bounded above. Now, $d(t, u)+d(t, t \vee v)+d(t, t \vee w)<n$, which is in conflict with (3). Therefore $n=3$, that is, $u, v, w$ cover $a$. Then the median $x$ of the vertices $u \vee v, u \vee w, v \vee w$ in $C(P)$ must be an upper bound of $u, v, w$ in the semilattice $P$ (see Figure 3), contrary to the assumption. Hence $P$ is a median semilattice.

\section{REFERENCES}

1. L. Alvarez, Undirected graphs realizable as graphs of modular lattices, Canad. J. Math. 17 (1965), 923-932.

2. S. P. Avann, Metric ternary distributive semi-lattices, Proc. Amer. Math. Soc. 12 (1961), 407-414.

3. H.-J. Bandelt and J. Hedliková, Median algebras, Discrete Math. 45 (1983), 1-30.

4. D. Duffus and I. Rival, Graphs orientable as distributive lattices, Proc. Amer. Math. Soc. 88 (1983), 197-200.

5. H. M. Mulder and A. Schrijver, Median graphs and Helly hypergraphs, Discrete Math. 25 (1979), 41-50.

6. M. Sholander, Medians, lattices, and trees, Proc. Amer. Math. Soc. 5 (1954), 808-812.

Department of Mathematics, Universität Oldenburg, D - 2900 Oldenburg, Federal Republic OF GERMANY 